\title{
The Gauss-Lucas Theorem for Regular Quaternionic Polynomials
}

\author{
Fabio Vlacci
}

\begin{abstract}
In this paper we extend the classical Gauss-Lucas Theorem from the setting of complex polynomials to the case of regular polynomials of quaternionic variables.
\end{abstract}

Mathematics Subject Classification (2000). Please provide some suitable AMS MSC 2010 subject codes.

Keywords. Please provide some suitable keywords.

\section{Introduction}

After Hamilton's discovery of quaternions, the strange new phenomena which take place in this non-commutative algebra attracted the interests of many mathematicians. For instance, in the study of quaternions from the algebraic point of view, it immediately turned out that, in general, the Fundamental Theorem of Algebra fails to be valid for quaternionic polynomials, as shown in the following example:

Example 1.1. For any $n \in \mathbb{N}$ and for any quaternion $q$, the polynomial

$$
a q^{n}-q^{n} a+1
$$

(with coefficient the quaternion $a$ ) has real part identically equal to 1 .

From the analytic point of view, the richness of the theory of holomorphic functions of one complex variable, along with motivations from physics, aroused interest in a theory of quaternion-valued functions of a quaternionic variable. In fact, several interesting theories have been introduced in the last century. The most famous is the one due to Fueter, [5, 6] of the mid 1930s; the basic results of this theory are accurately summarized in [22]. Recent work on Fueter-regularity includes $[2,15]$ and references therein. In the same years (see [17, 18]) there are the

This work is partially supported by G.N.S.A.G.A. of the I.N.D.A.M. and by M.I.U.R.. 
first attempts to find techniques to calculate roots of a class of quaternionic polynomials whereas an extensive description of the algebraic properties of polynomials in a non commutative setting can be found in [16].

A different theory of quaternion-valued functions of one quaternionic variable has been recently proposed by G. Gentili and D. C. Struppa [12]. The theory is based on a definition of regularity for quaternionic functions inspired by C. G. Cullen [4]. Several basic results of the theory are proven in [12], including the Cullen-regularity of quaternion power series and some nice properties of their zeroes. The study of the zero-sets of regular funcions has been further developed in $[9,24]$; in this paper we apply specific techniques from the theory of regular functions and establish a generalization of the Gauss-Lucas Theorem for quaternionic polynomials.

\section{Basic preliminary results for complex polynomials}

In this section we will shortly review well-known results ${ }^{1}$ for polynomials whose coefficients belong to $\mathbb{C}$ (the field of complex numbers) usually called complex polynomials. If $p$ is a complex polynomial, the set of all zeroes of $p$ will be denoted by $Z_{p}$ and also called the zero set of $p$.

If $p$ is considered as the holomorphic function $z \mapsto p(z)$, then we define $p^{\prime}$ to be the (complex) derivative of $p$. We recall that, by the celebrated Fundamental Theorem of Algebra, any non-constant complex polynomial has a root in $\mathbb{C}$ and that any zero of the (complex) polynomial $p^{\prime}$ is called critical point of $p$. The following geometric definition turns out to be very useful.

Definition 2.1. Given a subset $U$ of $\mathbb{C}$, we denote by $\mathcal{K}(U)$ the intersection of all convex sets which contain $U$. Clearly the set $\mathcal{K}(U)$ is convex. It is also called the convex hull of $U$.

A geometrical description of critical points in terms of roots of a complex polynomial $p$ is contained in the famous

Theorem 2.2 (Gauss-Lucas). Let p be a complex polynomial. The convex hull $\mathcal{K}\left(Z_{p}\right)$ of the zero-set of $p$ also contains the critical points of $p$; in particular,

$$
\mathcal{K}\left(Z_{p^{\prime}}\right) \subseteq \mathcal{K}\left(Z_{p}\right)
$$

The assertion of the previous theorem is equivalent to

Proposition 2.3. Every critical point $\xi$ of the complex polynomial $p$ is a convex linear combination of all zeroes of $p$, namely, if $p(z)=c \prod_{j=1}^{k}\left(z-z_{j}\right)^{m_{j}}$, then

$$
\xi=\sum_{j=1}^{k} \lambda_{j} z_{j}
$$

\footnotetext{
${ }^{1}$ We refer the interested reader to [20] for a more detailed introduction to the subject and for
} the proofs of the results stated in this section. 
with $\lambda_{j} \in \mathbb{R}, \lambda_{j} \geq 0 \forall j=1, \ldots, k$ such that $\sum_{j=1}^{k} \lambda_{j}=1$.

One of the advantages of Proposition 2.3 (compared to Theorem 2.2) is that it enlights the role played by the zeroes of $p$ for the determination of critical points of $p$.

\section{The Gauss-Lucas Theorem for regular polynomials in $\mathbb{H}$}

Denote by $\mathbb{S}$ the two-dimensional sphere of quaternion imaginary units: $\mathbb{S}=\{q \in$ $\left.\mathbb{H}: q^{2}=-1\right\}$. For any imaginary unit $I \in \mathbb{S}$, let $L_{I}=\mathbb{R}+I \mathbb{R}$ be the complex line through 0,1 and $I$. Any quaternion $q$ which is not real uniquely determines two real numbers $x$ and $y$ (with $y>0$ ) and an imaginary unit $I$ such that $q=x+I y$.

Definition 3.1. Let $\Omega \subseteq \mathbb{H}$ be a domain in $\mathbb{H}$; we say that $\Omega$ is

- a slice domain if $\Omega \cap \mathbb{R}$ is non-empty and if $L_{I} \cap \Omega$ is a domain in $L_{I}$ for all $I \in \mathbb{S}$;

- an axially symmetric domain if, for all $x+I y \in \Omega$, the whole 2 -sphere $x+\mathbb{S} y$ is contained in $\Omega$.

If $V$ is a subset of $\mathbb{H}$, then the set

$$
\widetilde{V}=\bigcup_{x+I y \in V} x+\mathbb{S} y
$$

is called the (axially) symmetric completion of $V$.

Clearly the unit ball $\mathbb{B} \subset \mathbb{H}$ is the first example of an axially symmetric slice domain and, in general, the axially symmetric slice domains turn out to be the natural domains of definition for an interesting class of functions which are currently under investigation and known as slice-regular or simply regular functions (see $[3,12])$.

Definition 3.2. Let $\Omega$ be a domain in $\mathbb{H}$ and let $f: \Omega \rightarrow \mathbb{H}$. Then $f$ is said to be slice-regular if, for all $I \in \mathbb{S}$, its restriction $f_{I}$ along $L_{I}$ has continuous partial derivatives and the function $\bar{\partial}_{I} f: \Omega \cap L_{I} \rightarrow \mathbb{H}$ defined by

$$
\bar{\partial}_{I} f(x+I y)=\frac{1}{2}\left(\frac{\partial}{\partial x}+I \frac{\partial}{\partial y}\right) f_{I}(x+I y)
$$

vanishes identically.

With the notations $\Omega_{I}=\Omega \cap L_{I}$ and $f_{I}=f_{\left.\right|_{\Omega_{I}}}$, we may refer to the vanishing of $\bar{\partial}_{I} f$ by saying that the restriction $f_{I}$ is holomorphic on $\Omega_{I}$. From now on we will refer to these functions simply just as regular functions. As observed in [12], a quaternion power series $\sum_{n \in \mathbb{N}} q^{n} a_{n}$ with $a_{n} \in \mathbb{H}$ defines a regular function in its domain of convergence, which proves to be an open ball $B(0, R)=\{q \in \mathbb{H}:|q|<$ $R\}$. In the same paper, it is also proven that 
Theorem 3.3. A function $f: B=B(0, R) \rightarrow \mathbb{H}$ is regular if and only if there exist quaternions $a_{n} \in \mathbb{H}$ such that

$$
f(q)=\sum_{n \in \mathbb{N}} q^{n} a_{n}
$$

for all $q \in B$. In particular, if $f$ is regular $f \in C^{\infty}(B)$.

The zero-set of a regular function is a very interesting geometric object (see $[3,9,12])$.

Theorem 3.4 (Structure of the Zero Set). Let $f: B(0, R) \rightarrow \mathbb{H}$ be a regular function and suppose $f$ does not vanish identically. Then the zero set of $f$ consists of isolated points or isolated 2-spheres of the form $S=x+y \mathbb{S}$, for $x, y \in \mathbb{R}$. In particular, the set of zeroes of a regular function whose coefficients are all in $L_{I}$ are either sphericals or they are isolated points contained in $L_{I}$.

The fact that a regular function $f$ has all the coefficients in $L_{I}$ has the important geometrical meaning that $f$ maps $L_{I}$ into itself; if the same happens for at least two different slices $L_{I}$ and $L_{J}$, then necessarily all the coefficients of $f$ are real and hence $f$ maps any slice $L_{I}$ into itself (see [12]).

Definition 3.5. Let $f(q)=\sum_{n=0}^{+\infty} q^{n} a_{n}$ and $g(q)=\sum_{n=0}^{+\infty} q^{n} b_{n}$ be given quaternionic power series with radii of convergence greater than $R$. We define the regular product of $f$ and $g$ as the series $f * g(q)=\sum_{n=0}^{+\infty} q^{n} c_{n}$, whose coefficients $c_{n}=\sum_{k=0}^{n} a_{k} b_{n-k}$ are obtained by discrete convolution from the coefficients of $f$ and $g$.

The regular product of $f$ and $g$ has a series expansion with radius of convergence greater than $R$. It can be easily proven that the regular multiplication $*$ is an associative, non-commutative operation and that (see [9])

$$
f * g(q)=f(q) g\left(f(q)^{-1} q f(q)\right) .
$$

Theorem 3.6. Let $f(q)=\sum_{n=0}^{+\infty} q^{n} a_{n}$ be a given quaternionic power series with radius of convergence $R$ and let $\alpha \in B(0, R)$. Then $f(\alpha)=0$ if and only if there exists a quaternionic power series $g$ with radius of convergence $R$ such that

$$
f(q)=(q-\alpha) * g(q) .
$$

This result (whose proof can be found in [9]) would of course be uninteresting if the other zeroes of $f$ did not depend on the zeroes of $g$. Fortunately, this is not the case: the zeroes of a regular product $f * g$ are strongly related with those of $f$ and $g$, as shown by the following (see [9])

Theorem 3.7 (Zeroes of a regular product). Let $f, g$ be given quaternionic power series with radii greater than $R$ and let $\alpha \in B(0, R)$. Then $f * g(\alpha)=0$ if and only if $f(\alpha)=0$ or $f(\alpha) \neq 0$ and $g\left(f(\alpha)^{-1} \alpha f(\alpha)\right)=0$. 
Definition 3.8. Let $f(q)=\sum_{n=0}^{+\infty} q^{n} a_{n}$ be a given quaternionic power series with radius of convergence $R$. We define the regular conjugate of $f$ as the series $f^{c}(q)=$ $\sum_{n=0}^{+\infty} q^{n} \bar{a}_{n}$

We remark that $f^{c}$ also has radius $R$ and, in general, if $h=f * g$, then $h^{c}=g^{c} * f^{c}$. If we define $f^{s}=f * f^{c}=f^{c} * f$, then $f^{s}$ also has radius $R$. Notice furthermore that the coefficients of $f^{s}$ are all real and that if the coefficients of $f$ are all real, then simply $f^{s}=f^{2}$.

Something more precise can be actualy proven about the zeroes of $f$, of $f^{c}$ and of $f^{s}$ (see [9]).

Proposition 3.9. Let $f$ be a given quaternionic power series with radius of convergence $R$ and let $x, y \in \mathbb{R}$ be such that $S=x+y \mathbb{S} \subseteq B(0, R)$. The zeroes of $f$ in $S$ are in one-to-one correspondence with those of $f^{c}$.

Proposition 3.10. Let $f(q)=\sum_{n=0}^{+\infty} q^{n} a_{n}$ be a given quaternionic power series with radius of convergence $R$. If $\alpha=x_{0}+I_{0} y_{0}$ (with $x_{0}, y_{0} \in \mathbb{R}, I_{0} \in \mathbb{S}$ ) is such that $f(\alpha)=f\left(x_{0}+I_{0} y_{0}\right)=0$, then $f^{s}\left(x_{0}+L y_{0}\right)=0$ for all $L \in \mathbb{S}$.

Symmetrization allows us indeed to transform any zero into a "spherical" zero and these zeroes can not accumulate unless the regular function is constant: indeed if the spherical zeroes accumulate, then the same happens in each complex line $L_{I}$ and this is impossible for the Identity Principle, unless the regular function is constant.

From now on we will focus our attention to the case of regular polynomials of quaternionic variable, i.e., according to Theorem 3.3, polynomials with coefficients in $\mathbb{H}$ of the form

$$
P(q)=q^{n} a_{n}+q^{n-1} a_{n-1}+\ldots+q a_{1}+a_{0} .
$$

First of all, for regular polynomials the Fundamental Theorem of Algebra holds true (see [14, 17, 18]); furthermore, the following result is proved in [13].

Theorem 3.11. Let $P$ be a regular polynomial of degree $m$. Then there exist $p, m_{1}$, $\ldots, m_{p} \in \mathbb{N}$, and $w_{1}, \ldots, w_{p} \in \mathbb{H}$, generators of the spherical roots of $P$, so that

$$
P(q)=\left(q^{2}-2 q R e\left(w_{1}\right)+\left|w_{1}\right|^{2}\right)^{m_{1}} \cdots\left(q^{2}-2 q R e\left(w_{p}\right)+\left|w_{p}\right|^{2}\right)^{m_{p}} Q(q),
$$

where $R e\left(w_{i}\right)$ denotes the real part of $w_{i}$ and $Q$ is a regular polynomial with coefficients in $\mathbb{H}$ having only non spherical zeroes. Moreover, if $n=m-2\left(m_{1}+\cdots+m_{p}\right)$ there exist a constant $c \in \mathbb{H}, t$ distinct 2 -spheres $S_{1}=x_{1}+y_{1} \mathbb{S}, \ldots, S_{t}=x_{t}+y_{t} \mathbb{S}, t$ integers $n_{1}, \ldots, n_{t}$ with $n_{1}+\cdots+n_{t}=n$, and (for any $i=1, \ldots, t$ ) $n_{i}$ quaternions $\alpha_{i j} \in S_{i}, j=1, \ldots, n_{i}$, such that

$$
Q(q)=\left[\prod_{i=1}^{t} \prod_{j=1}^{n_{i}}\left(q-\alpha_{i j}\right)\right] c
$$


where $\mathbb{W}$ is the analogous of $\prod$ in the case of $*$-product.

Notice that, even though $\alpha_{i j} \in S_{i}$ is not necessarily a root of the polynomial $P$, the polynomial $P$ certainly has an isolated zero on the sphere $S_{i}=x_{i}+y_{i} \mathbb{S}$.

Definition 3.12. Let $P$ be a regular polynomial with quaternionic coefficients. If $x+y \mathbb{S}$ is a spherical root of $P$, its multiplicity is defined as two times the largest integer $m$ for which it is possible to factor $\left(q^{2}-2 q x+\left(x^{2}+y^{2}\right)\right)^{m}$ from $P$. On the other hand, we say that $\alpha \in \mathbb{H}$ has multiplicity $k$ as an (isolated) root for $P$ if, in the factorization (6), there are exactly $k$ quaternions $\alpha_{i j}$ which lie on the sphere $S_{\alpha}$.

Remark 3.13. Since the coefficients of $P^{s}$ are real, then $\left.P^{s}\right|_{L_{I}}$ is a complex polynomial and maps $L_{I}$ into itself; therefore the Gauss-Lucas Theorem can be applied to the restriction along $L_{I}$ of the symmetrization $P^{s}$ of a polynomial $P$; and this can be done for any $I \in \mathbb{S}$. Notice furthermore that, according to Proposition 3.4 and the definition of symmetrized polynomial, if $\operatorname{deg} P=n$, then the zero-set of $P^{s}$ when restricted along $L_{I}$ is a set of (at most) $2 n$ points; these points, when non-real, are symmetric in pairs, with respect to the real axis. And this geometric configuration of the zero-set along $L_{I}$ is exactly the same for the restriction of $P^{s}$ along any $L_{I}$ with $I \in \mathbb{S}$.

Thus, recalling Proposition 3.10, we have

Proposition 3.14. Any critical point of a regular polynomial $P$ with quaternionic coefficients is in (the axially symmetric completion of) the convex hull $\mathcal{K}\left(Z_{P s}\right)$ of the zero set of $P^{s}$.

Proof. Consider

$P(q)=\left(q^{2}-2 q R e\left(w_{1}\right)+\left|w_{1}\right|^{2}\right)^{m_{1}} \cdots\left(q^{2}-2 q R e\left(w_{p}\right)+\left|w_{p}\right|^{2}\right)^{m_{p}}\left[\left.\right|_{i=1} ^{t}||_{j=1}^{n_{i}}\left(q-\alpha_{i j}\right)\right] c ;$

then, after rearranging indexes, the zero-set of the restriction of $P^{s}$ along $L_{I}$ can be written as

$Z_{\left.P^{s}\right|_{L_{I}}}=\left\{w_{1}^{I}, \ldots, w_{p}^{I}, \overline{w_{1}^{I}}, \ldots, \overline{w_{p}^{I}}\right\} \cup\left\{\alpha_{1}^{I}, \ldots, \alpha_{t_{1}-1}^{I}, \overline{\alpha_{1}^{I}}, \ldots, \overline{\alpha_{t_{1}-1}^{I}}\right\} \cup\left\{\alpha_{t_{1}}^{I}, \ldots, \alpha_{t}^{I}\right\}$

where $\alpha_{s}^{I}$ is real for $t_{1} \leq s \leq t$. Thus, according to Proposition 2.3 applied to $\left.P^{s}\right|_{L_{I}}$, any critical point $\xi$ of $\left.P^{s}\right|_{L_{I}}$ can be written in the following way,

$$
\xi=\sum_{j=1}^{p}\left(\lambda_{j} w_{j}^{I}+\lambda_{j}^{\prime} \overline{w_{j}^{I}}\right)+\sum_{k=1}^{t_{1}-1}\left(\mu_{k} \alpha_{k}^{I}+\mu_{k}^{\prime} \overline{\alpha_{k}^{I}}\right)+\sum_{s=t_{1}}^{t} \tau_{s} \alpha_{s}^{I}
$$

with $\lambda_{j}, \lambda_{j}^{\prime}, \mu_{k}, \mu_{k}^{\prime}, \tau_{s} \in \mathbb{R}, \lambda_{j} \geq 0, \lambda_{j}^{\prime} \geq 0 \forall j=1, \ldots, p, \mu_{k} \geq 0, \mu_{k}^{\prime} \geq 0$ $\forall k=1, \ldots, t_{1}-1, \tau_{s} \geq 0 \forall s=t_{1}, \ldots, t$ and such that $\sum_{j=1}^{k}\left(\lambda_{j}+\lambda_{j}^{\prime}\right)+\sum_{k=1}^{t_{1}-1}\left(\mu_{k}+\right.$ $\left.\mu_{k}^{\prime}\right)+\sum_{s=t_{1}}^{t} \tau_{s}=1$. 
We can now provide a different, more direct, proof of the previous result in the case of quaternionic polynomials with non-spherical zeroes only. Given such a regular polynomial $P$ with quaternionic coefficients and degree $d$, then there exist $d$ quaternions $\alpha_{j}$ and a constant $c$ such that

$$
P(q)=\left(q-\alpha_{1}\right) *\left(q-\alpha_{2}\right) * \ldots\left(q-\alpha_{d}\right) c ;
$$

consider the associated polynomial

$$
\widetilde{P}(q)=\left(q-\alpha_{1}\right) *\left(q-\widetilde{\alpha_{2}}\right) * \ldots\left(q-\widetilde{\alpha_{d}}\right)
$$

where if $\alpha_{k}=x_{k}+I_{k} y_{k}$, the $d-1$ quaternions $\widetilde{\alpha_{2}}, \ldots, \widetilde{\alpha_{d}}$ are uniquely determined by the conditions

$$
\widetilde{\alpha_{j}}=x_{j}+I_{1} y_{j} \quad \forall j \text {, such that } 2 \leq j \leq d .
$$

The axially symmetric completion $\widehat{Z}_{P}$ of the zero-set $Z_{P}$ of $P$ clearly coincides with the axially symmetric completion $\widehat{Z}_{\widetilde{P}}$ of the zero-set of $\widetilde{P}$. But since the regular polynomial $\widetilde{P}$ has all the coefficients in $L_{I_{\alpha_{1}}}$, the zero-set of $\widetilde{P}$ is contained in $L_{I_{\alpha_{1}}}$, since $P$ (and hence $\widetilde{P}$ ) has no spherical zeroes. Thus the set $\mathcal{Z}_{\widetilde{P}}$ of critical points of $\widetilde{P}$ is contained in the convex hull $\mathcal{K}\left(Z_{\widetilde{P}}\right)$ of the zero-set $Z_{\widetilde{P}}$ of $\widetilde{P}$ and hence the set $\mathcal{Z}_{P}$ of critical points of $P$ is contained in the axially symmetric completion $\widehat{\mathcal{K}\left(Z_{\widetilde{P}}\right)}$ of the convex hull of the zero-set of $\widetilde{P}$.

\section{References}

[1] L. Ahlfors, Complex analysis, McGraw-Hill, New York (1979).

[2] F. Colombo, I. Sabadini, F. Sommen, D.C. Struppa, Analysis of Dirac Systems and Computational Algebra, Birkhäuser, 2004.

[3] F. Colombo, G. Gentili, I. Sabadini, D. C. Struppa, Extension results for slice regular functions of a quaternionic variable. Adv. Math. 222 (2009), 1793-1808.

[4] C. G. Cullen, An integral theorem for analytic intrinsic functions on quaternions. Duke Math. J. 32 (1965), 139-148.

[5] R. Fueter, Die Funktionentheorie der Differentialgleichungen $\Delta u=0$ und $\Delta \Delta u=0$ mit vier reellen Variablen. Comment. Math. Helv. 7 (1934/5), 307-330.

[6] R. Fueter, Über die analytische Darstellung der regulären Funktionen einer Quaternionenvariablen. Comment. Math. Helv. 8 (1935/6), 371-378.

[7] G. Gentili, C. Stoppato, Power series and analyticity over the quaternions, Preprint, arXiv:0902.4679 [math.CV], (2009)

[8] G. Gentili, C. Stoppato, The open mapping theorem for quaternionic regular functions. Ann. Sc. Norm. Super. Pisa Cl. Sci. 5 (2009), 805-815.

[9] G. Gentili, C. Stoppato, Zeros of regular functions and polynomials of a quaternionic variable. Michigan Math. J. 56 (2008), 655-667.

[10] G. Gentili, C. Stoppato, D. C. Struppa, F. Vlacci, Recent developments for regular functions of a hypercomplex variable. Hypercomplex Analysis, Trends in Mathematics, 165-186, Birkhauser, Basel (2009). 
[11] G. Gentili, D. C. Struppa, A new approach to Cullen-regular functions of a quaternionic variable. C. R. Math. Acad. Sci. Paris 342 (2006), 741-744.

[12] G. Gentili, D. C. Struppa, A new theory of regular functions of a quaternionic variable. Adv. Math. 216 (2007), 279-301.

[13] G. Gentili, D. C. Struppa, On the multiplicity of the zeroes of polynomials with quaternionic coefficients, Milan J. Math., 76, (2008), 15-25.

[14] G. Gentili - D. C. Struppa - F. Vlacci, The Fundamental Theorem of Algebra for Hamilton and Cayley numbers, Math. Z., 259, (2008), 895-902.

[15] V.V. Kravchenko, M.V. Shapiro, Integral Representations for Spatial Models of Mathematical Physics, Pitman Research Notes in Mathematics Series, 351, Longman, Harlow, 1996.

[16] T. Y. Lam, A first course in noncommutative rings. Graduate Texts in Mathematics, 123, Springer-Verlag, New York, 1991.

[17] J. Niven Equations in quaternions, Amer. Math. Monthly 48, (1941), 654-661.

[18] J. Niven The roots of a quaternion,Amer. Math. Monthly 49, (1942), 386-388.

[19] A. Pogorui, M. V. Shapiro, On the structure of the set of zeros of quaternionic polynomials. Complex Variables Theory Appl. 49 (2004), no. 6, 379-389.

[20] Q.I. Rahman, G. Schmeisser, Analytic Theory of Polynomials Claredon Press, Oxford, (2002).

[21] C. Stoppato, Poles of regular quaternionic functions, to appear in Complex Var. Elliptic Equ.

[22] A. Sudbery, Quaternionic analysis. Math. Proc. Camb. Phil. Soc. 85 (1979), 199-225.

[23] I. Vignozzi, Funzioni Intere sui Quaternioni: Fattorizzazioni degli Zeri, Tesi di Laurea, Università di Firenze (2008).

[24] F. Vlacci, The Argument Principle for Quaternionic Regular Functions, to appear in Michigan Math. J.

Fabio Vlacci

Dipartimento di Matematica "U. Dini"

Università di Firenze

Viale Morgagni 67/A

50134 Firenze

Italy

e-mail: vlacci@math.unifi.it 